\title{
Research Article \\ Localized Spectral Analysis of Fluctuating Power Generation from Solar Energy Systems
}

\author{
Achim Woyte, ${ }^{1}$ Ronnie Belmans, ${ }^{2}$ and Johan Nijs ${ }^{2,3}$ \\ ${ }^{1} 3 E$ sa, Rue du Canal 61, 1000 Brussels, Belgium \\ ${ }^{2}$ Departement Elektrotechniek, Katholieke Universiteit Leuven, Kasteelpark Arenberg 10, 3001 Leuven, Belgium \\ ${ }^{3}$ Photovoltech sa, Grijpenlaan 18, 3300 Tienen, Belgium
}

Received 27 April 2006; Revised 20 December 2006; Accepted 23 December 2006

Recommended by Alexander Mamishev

\begin{abstract}
Fluctuations in solar irradiance are a serious obstacle for the future large-scale application of photovoltaics. Occurring regularly with the passage of clouds, they can cause unexpected power variations and introduce voltage dips to the power distribution system. This paper proposes the treatment of such fluctuating time series as realizations of a stochastic, locally stationary, wavelet process. Its local spectral density can be estimated from empirical data by means of wavelet periodograms. The wavelet approach allows the analysis of the amplitude of fluctuations per characteristic scale, hence, persistence of the fluctuation. Furthermore, conclusions can be drawn on the frequency of occurrence of fluctuations of different scale. This localized spectral analysis was applied to empirical data of two successive years. The approach is especially useful for network planning and load management of power distribution systems containing a high density of photovoltaic generation units.
\end{abstract}

Copyright (C) 2007 Achim Woyte et al. This is an open access article distributed under the Creative Commons Attribution License, which permits unrestricted use, distribution, and reproduction in any medium, provided the original work is properly cited.

\section{INTRODUCTION}

Most applications of wavelet decomposition in the field of electrical power engineering concern the analysis of load profiles $[1,2]$, the electrical power supply quality and its measurement [3-6], and also protection issues [7]. The primary objective in most of these applications is the isolation of transient phenomena from steady-state phenomena in the electricity grid, usually the fundamental 50 or $60 \mathrm{~Hz}$ component and its harmonics [7]. A new area of application is presented with the analysis of time series of solar radiation in order to quantify the intermittent power supplied by solar energy systems, mainly photovoltaics (PV). In this case, the power supply quality can be deteriorated as a consequence of power variations due to a varying cloud coverage of the sky. This leads to variable power output of the PV system, which introduces voltage dips to the distribution system. Typically these fluctuations persist seconds up to a fraction of an hour.

The intermittent nature of solar radiation is one of the drawbacks of the large-scale application of photovoltaics. With a high density of $\mathrm{PV}$ generation in a power distribution grid, irradiance fluctuations introduced by moving clouds can lead to unpredictable variations of node voltages and power and finally cause a breakdown of distribution grids.
Distribution system operators need tools for a realistic estimation of such disturbances, allowing to take adequate measures for grid reinforcement in time while avoiding too cautious and, therefore, cost-intensive measures. An analysis of fluctuations introduced by solar irradiance fluctuations must focus on their amplitude, persistence, and frequency of occurrence rather than their location in time. A tool that would allow the distribution system operator to stochastically assess these parameters would be of utmost practical utility.

The Fourier analysis cannot satisfactorily provide the necessary information since time series of solar irradiance exhibit no intraday periodicity. Instead, a localized spectral analysis based on wavelet bases is proposed. This analysis permits the decomposition of the fluctuating irradiance signal into a set of orthonormal subsignals. Each of them represents one specific scale of persistence of the fluctuation.

The objective of this study is to illustrate the application of localized spectral analysis in the field of solar energy meteorology, as a new tool for facilitating the integration of PV generation units in the power distribution systems of the future. Exemplary applications of the method to electric power systems have already been presented in $[8,9]$ whereas the meteorological conditions allowing the generalization of this method were explored in [10]. The present paper introduces 
the analysis of empirical time series derived from solar irradiance as realizations of a stochastic, locally stationary, wavelet process, following the approach proposed in [11-13]. The occurring fluctuations can be classified and treated per characteristic scale of persistence. As a result, fluctuation indices are derived for all characteristic scales, permitting conclusions on the characteristic fluctuation pattern at the specific site.

\section{PRECEDING STUDIES}

Two studies assessing cloud-induced power fluctuations in distribution grids with high PV connection density are presented in $[14,15]$. The examinations are based on the approximation of clouds by primitive geometries, moving over the area under examination with predefined wind speed and direction. Conclusions on the frequency of occurrence and duration of irradiance fluctuations have not been drawn.

In [16], the contours of clouds in inhomogeneous skies are modelled as fractals, taking into account the irregular shape and spatial distribution of clouds. Based on this model, time series of solar irradiance have been synthesized and applied to extended power-flow studies. Within this approach, the fractal dimension is a measure for the cloud-induced variability of solar radiation. However, further steps regarding the classification of cloudy sky conditions by means of this approach have not yet been published.

A statistical approach is applied in [17]. There, time series of solar irradiance are described by their "fluctuation factor," being defined as the root mean square ( $\mathrm{rms}$ ) value of the high-pass filtered time series of solar irradiance, recorded during two hours around noon. The authors propose the power spectral density (PSD) of the irradiance time series as a potential tool for the analysis of cloud fields, without yet further elaborating the approach. However, a main drawback of the PSD approach seems to be the obvious lack of second-order autocorrelation in time series of the clearness index.

The proposed analysis of short fluctuations in solar irradiance by means of localized spectral analysis can combine advantages of $[16,17]$. On the one hand, similar to fractal cloud patterns [16], the approach allows the analysis of all scales of fluctuation from very short variations as they appear close to the edge of a cloud, up to long fluctuations between clouds. On the other hand, the wavelet approach allows quantifying the power content of the fluctuating signal, similar to the fluctuation factor in [17]. Moreover, due to its good time-frequency localization, the wavelet approach allows a meaningful decomposition of the signal's power content, corresponding to the persistence of the occurring fluctuation. Finally, unlike many other approaches described in the literature, the wavelet approach is mathematically sound.

\section{BACKGROUND}

\subsection{Solar irradiance signals}

The solar irradiance $G(t)$ received by an arbitrarily oriented surface as a function of time can be decomposed into a deterministic and a stochastic part according to

$$
G(t)=I_{0} E_{0}(t) \cos \gamma_{i}(t) k(t)
$$

with

(i) $I_{0}=1367 \mathrm{~W} / \mathrm{m}^{2}$ the solar constant, defined as the long-term average intensity of solar radiation as received outside the earth's atmosphere,

(ii) $E_{0}(t)$ the eccentricity correction factor, compensating for periodic annual variations of the earth's orbit,

(iii) $\gamma_{i}(t)$ the angle of incidence of the sun rays on an arbitrarily oriented surface at a given geographical position,

(iv) $k(t)$ the instantaneous clearness index [18].

For a receiver with arbitrarily oriented surface, $E_{0}$ and $\gamma_{i}$ only depend on astronomical relationships and can be analytically determined for each instant in time throughout the year. The clearness index $k$ accounts for all meteorological influences, mainly being the stochastic parameters atmospheric turbidity and moving clouds. It is independent of all astronomical relationships. The mean value of the clearness index over a period of time is denoted as $\bar{k}$. The sampling period $\Delta T$ for an analysis of cloud-induced fluctuations should be no longer than eight seconds in order to account for more than $98 \%$ of the signal's power content [10].

Figure 1(a) shows the solar irradiance on a slightly overcast summer day with $\bar{k}=0.73$ as a function of time, sampled as 5-second average values. The corresponding time series of the clearness index in Figure 1(b), calculated by means of (1), exhibits no significant trend and its fluctuations appear to be randomly distributed in time. A closer view on the short-time behavior of this signal in Figure 1(c) displays the influence of passing clouds, when the radiation sensor receives only diffuse irradiance, but no beam irradiance directly from the sun. This bimodal, almost binary, behavior of the instantaneous clearness index with distinct "clear" and "cloudy" states is well known in the field of solar energy meteorology $[14,19,20]$. Apparently, time series of the instantaneous clearness index can be characterized as a signal of randomly distributed squares of variable pulse width, superposed by higher frequency noise, mainly, but not exclusively, occurring at the transitions between clear and cloudy states.

The cumulative frequency distribution of the clearness index, averaged over one hour and more, can be described by Boltzmann statistics [20-24]. Remarkably, for any specified mean value $\bar{k}$ during the period under study, the probability distribution of $k$ is virtually independent of the season and the geographical position. With some limitations, this also holds for the instantaneous clearness index, with its frequency distribution defined as a superposition of different Boltzmann distributions, accounting for the sharp transition between clear and cloudy states and the comparably scarce occurrence of intermediate ones [10, 20]. Autocorrelation analysis of time series of the instantaneous clearness index returned first-order autocorrelation coefficients with sufficiently low variance for clearness index values sampled as 5-minute averages and longer. For shorter averaging times, 


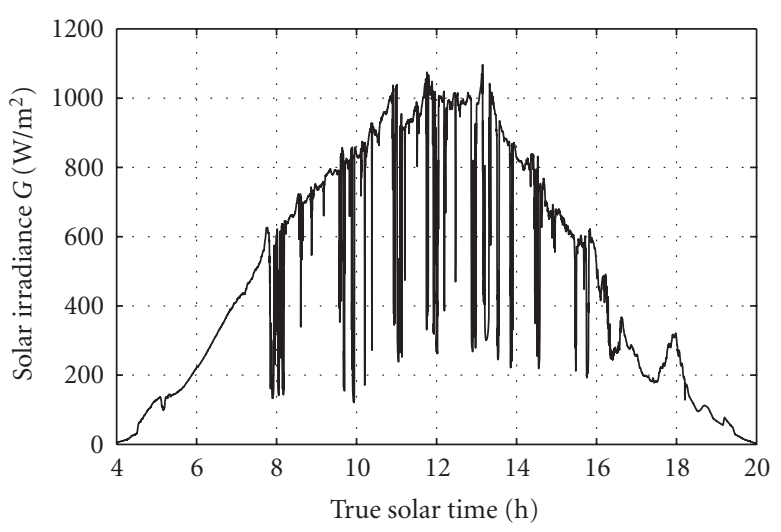

(a) Global irradiance on the horizontal plane.

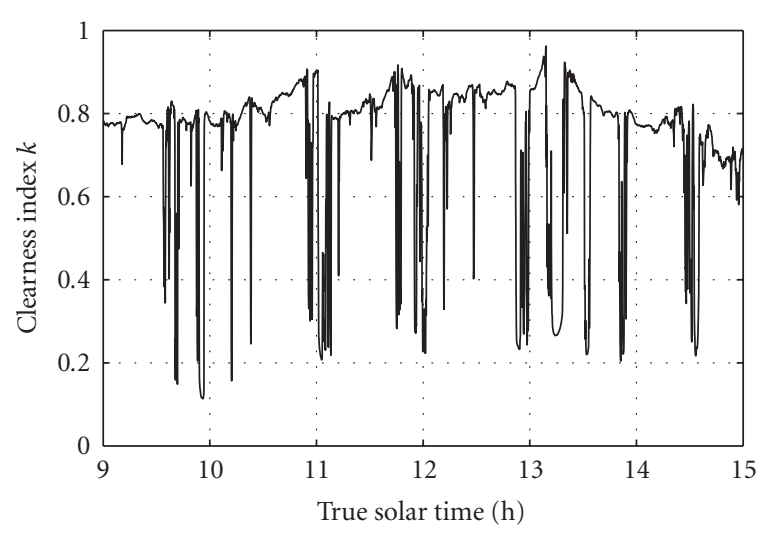

(b) Clearness index during 6 hours around noon.

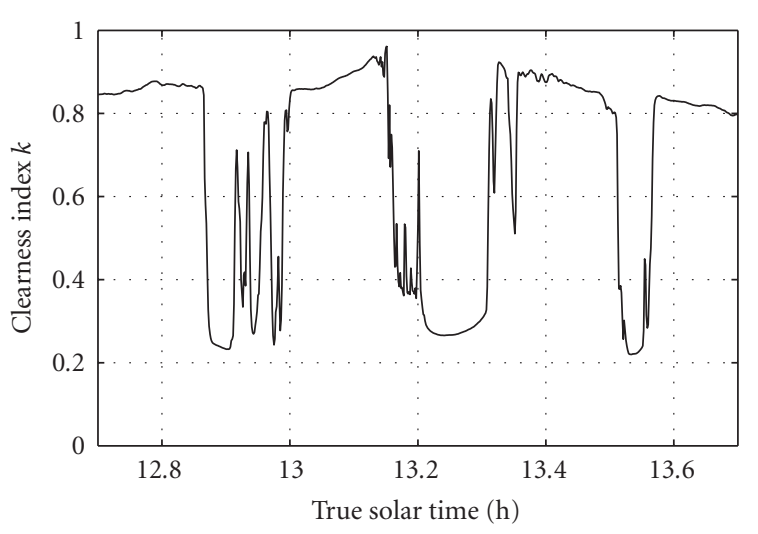

(c) Clearness index zoomed in on 1 hour.

FIgURE 1: Global irradiance and clearness index on a slightly clouded summer day (June 19, 2001) with daily mean clearness in$\operatorname{dex} \bar{k}=0.73$.

no significant autocorrelation coefficients could be identified [19].

While the frequency distribution of the instantaneous clearness index is well determined, due to the obvious lack of periodicity, no significant second-order autocorrelation can be identified in recorded time series of the instantaneous clearness index. Autocorrelation analysis of time series of one-second average values measured in Leuven, Belgium, returned no characteristic periodicity with regard to cloud coverage. Hence, stochastic modelling of time series of the instantaneous clearness index, as required for forecasting, is almost impossible. Nevertheless, methods for the analysis of time series of the clearness index, as realizations of the underlying random process, are of even greater importance.

\subsection{Localized spectral analysis}

The daily time series of the instantaneous clearness index are interpreted as realizations of a stochastic, locally stationary wavelet (LSW) process. The power content of such a process, decomposed per wavelet scale, at each particular time is determined by its local spectral density (LSD) with, as an estimator, the wavelet periodogram of the sequence analyzed. A number of practical examples of wavelet periodogram analysis of empirical signals has already been provided in [11], and the underlying process model was refined in $[12,13]$.

Wavelet periodogram analysis is based on the socalled dyadic, undecimated, or stationary wavelet transform (SWT). Unlike the more common discrete wavelet transform, the SWT contains redundancy, but it features the advantage of time invariance, which is essential for the analysis of the stochastic time series under consideration [2527]. For a discrete sequence $x=\{x[n]\}$ of length $N$, with $n=0,1, \ldots, N-1$, the SWT is calculated from

$$
\begin{aligned}
& \mathcal{D}_{j}(x)[v]=\sum_{n=0}^{N-1} x[n] \frac{1}{2^{j / 2}} \psi^{*}\left(\frac{n-v}{2^{j}}\right), \\
& \mathcal{A}_{j}(x)[v]=\sum_{n=0}^{N-1} x[n] \frac{1}{2^{j / 2}} \phi^{*}\left(\frac{n-v}{2^{j}}\right),
\end{aligned}
$$

where the function $\psi$ is referred to as the mother wavelet with $\phi$ its corresponding scaling function [28]. The asterisk (*) indicates complex conjugation. The length- $N$ sequences $\mathscr{D}_{j}=\left\{\mathscr{D}_{j}(x)[\nu]\right\}$ and $\mathscr{A}_{j}=\left\{\mathscr{A}_{j}(x)[\nu]\right\}$ are referred to as "detail $j$ " and "approximation $j$," respectively.

Since the SWT contains redundancy, its inverse is not unique, although, for practical application, it can be approximated by the average over all existing inverse transforms. When $\psi$ is an orthonormal wavelet base, the SWT still ensures orthogonality between scales, and, with proper normalization, Parseval identity is maintained:

$$
\|x\|_{2}^{2}=\frac{1}{2^{j_{0}}}\left\|\mathcal{A}_{j_{0}}\right\|_{2}^{2}+\sum_{j=1}^{j_{0}} \frac{1}{2^{j}}\left\|\mathcal{D}_{j}\right\|_{2}^{2}, \quad j_{0} \leq \log _{2} N \in \mathbb{N},
$$

hence, the set of sequences $\mathcal{W}=\left\{\mathcal{A}_{j_{0}}, \mathscr{D}_{1}, \mathscr{D}_{2}, \ldots, \mathscr{D}_{j_{0}}\right\}$ is a complete representation of the original sequence $x$.

As an estimator of the LSD of the LSW process under consideration, the wavelet periodogram $\ell$ can be calculated from the SWT of the empirical time series. With the normalization as in (3), the values of $\ell=\left\{\left\{I_{j}[\nu]\right\}\right\}$ are calculated from

$$
I_{j}[\nu]=\frac{1}{2^{j}}\left|\mathscr{D}_{j}(x)[\nu]\right|^{2} .
$$




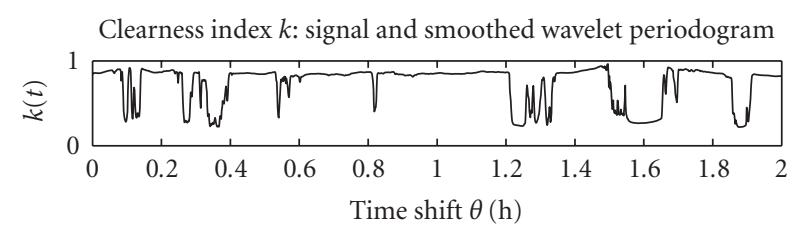

(a)

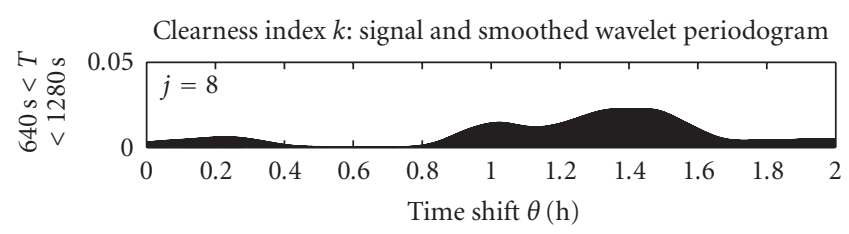

(c)

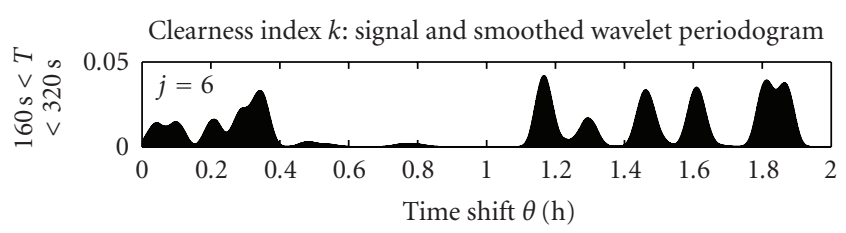

(e)

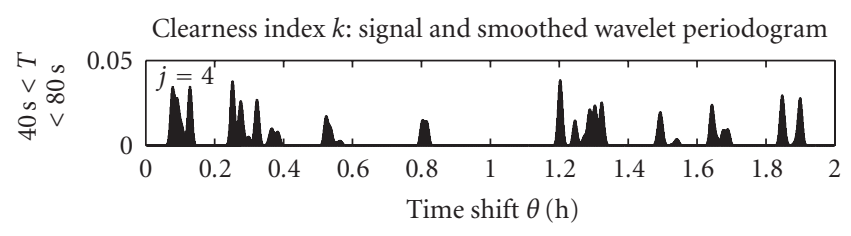

(g)

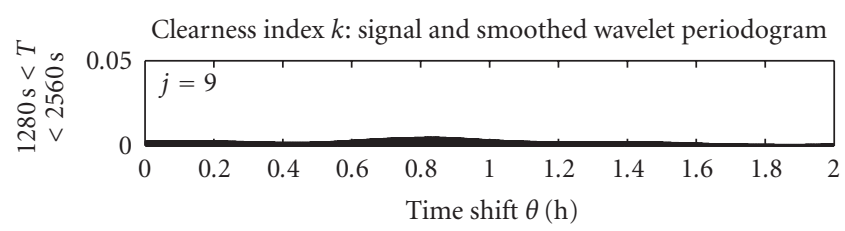

(b)

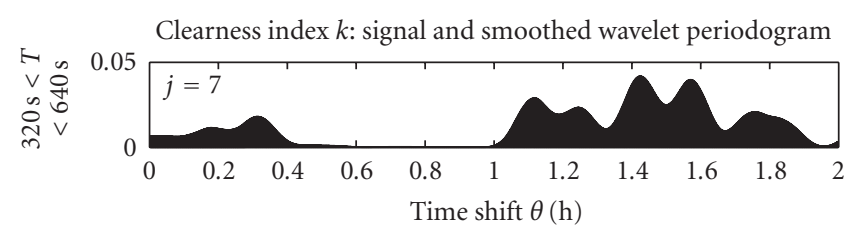

(d)

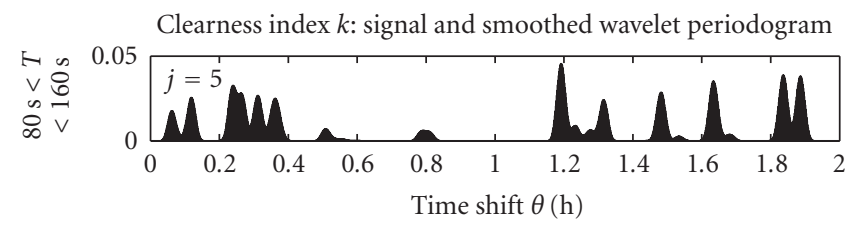

(f)

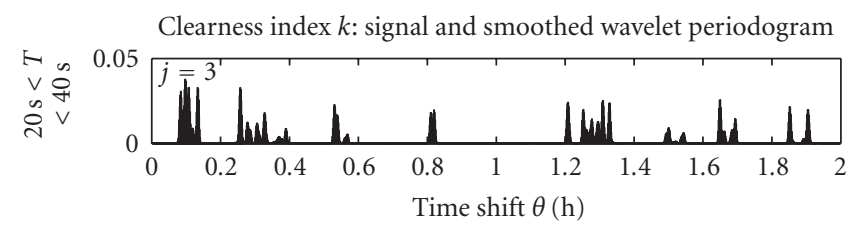

(h)

FIGURE 2: Smoothed wavelet periodogram of a time series of the instantaneous clearness index; $\theta=\nu \Delta T$ with $\Delta T=5$ seconds, $j_{0}=12$, $N=4096$, shown are only $j=3$ up to 9 during 2 hours, $\psi$ : Haar wavelet.

In an additional step, following the analysis from [11], the sequences $\left\{I_{j}[\nu]\right\}$ are smoothed in order to eliminate higher-frequency components introduced by the square in (4). This is done by means of a stationary wavelet filter with the same mother wavelet as above. Each sequence $\left\{I_{j}[\nu]\right\}$ is transformed by means of an SWT and back, after having set to zero all coefficients at levels of scale smaller than $j$. The smoothed wavelet periodogram is denoted as $\ell^{\prime}=\left\{\left\{I_{j}^{\prime}[\nu]\right\}\right.$.

\section{APPLICATION TO THE INSTANTANEOUS CLEARNESS INDEX}

The smoothed wavelet periodogram is a measure for the local power content of the clearness index signal for all dyadic scales $2^{j}$, and it is variable in time with the time shift $\theta$. Figure 2 shows a typical two-hour clearness index signal and its wavelet periodogram for a number of significant scales.

The Haar wavelet has been chosen as the mother wavelet. Due to its rectangular shape, the Haar wavelet corresponds very well to the bimodal character of the clearness index. Fluctuations of the clearness index (upper graph) are associated with local maxima on that scale, corresponding to the length of the particular fluctuation. Although some leakage between scales cannot be entirely prevented, the decomposition based on the Haar wavelet exhibits a close correspondence between the persistence of a fluctuation and the scale of the wavelet periodogram on which the associated maximum occurs.

For example, the "dip" with approximately 200-second persistence, occurring around $\theta=1.9$ hours, causes a maximum on the scale with $j=6$, with some leakage to the neighbouring scales. Conversely, the much shorter dip, occurring shortly after $\theta=0.8$ hour, mainly affects the scales with $j=3$ and 4. Equation (3) is still valid after smoothing and the sum over all time-integrated scales of the periodogram equals the energy content of the analyzed clearness index signal.

For the characterization of the signal's mean power content and the energy associated with a fluctuation on the different scales, at this place, the "fluctuation power index" and "fluctuation energy index" are introduced. The fluctuation power index $c f_{p}$ is defined as the mean spectral density of a sequence $x$ as a function of the level of scale $j$ :

$$
c f_{p}[j]=\frac{1}{N} \sum_{\nu=0}^{N-1} I_{j}[\nu]
$$


The fluctuation power index represents the mean square value, thus, the average power, of all fluctuations in the sequence $x$ on the particular scale.

With the characteristic persistence of a fluctuation that is associated with the level of scale $j$ being defined as

$$
S_{j}=2^{j-1}
$$

the fluctuation energy index $c f_{e}$ is calculated

$$
c f_{e}[j]=S_{j} c f_{p}[j] .
$$

The fluctuation energy index is a measure for the energy that is typically bound and freed again during a signal fluctuation of the persistence $2 S_{j}$.

Applied to time series of the instantaneous clearness in$\operatorname{dex}, c f_{p}$ as a function of $S_{j}$ is a measure of the amplitude and frequency of power flow fluctuations of a given persistence, introduced by PV generation.

It is important to note that the terms power and energy in this context describe mathematical concepts rather than physical quantities. In electrical engineering, the mean square value of a signal is usually interpreted as its power [29]. Mathematicians would rather talk about variance [13]. However, in thermodynamic terms, the instantaneous clearness index already is proportional to solar power. Its fluctuation power index over all scales, therefore, represents the square of solar power. Accordingly, the fluctuation energy index over all scales represents the integrated square of solar power over the time of persistence and not the integrated solar power. Here, $c f_{e}$ has mainly been developed for reasons of completeness but it will not further be applied in this analysis.

\section{RESULTS AND PRACTICAL APPLICATION}

\subsection{Statistical interpretation}

The question arises whether for a given climate the stochastic moments of the LSD of the clearness index can be determined with a sufficiently low variance. If this is the case, conclusions become possible, regarding the estimated frequency of occurrence of fluctuations along with their amplitude for each particular scale. Doubtlessly, for substantiated conclusions, a thorough quantitative analysis is required, based on an extended set of empirical data, measured over several years on different sites. Nevertheless, first results based on a limited set of data indicate that regularities in the frequency distribution of $c f_{p}$ exist.

Smoothed wavelet periodograms and fluctuation indices have been calculated for time series of the clearness index from 721 sample days recorded during roughly two years in Leuven, Belgium (situated $4.7^{\circ} \mathrm{E}, 50.9^{\circ} \mathrm{N}, 30 \mathrm{~m}$ a.s.l., moderate maritime climate). The time series have been chosen symmetrically around solar noon containing 4096 equidistant samples, each with a sampling period $\Delta T=5$ seconds. The time series have been grouped in seven classes according to their mean clearness index $\bar{k}$, and annual mean values of $c f_{p}$ have been calculated for each class of $\bar{k}$.

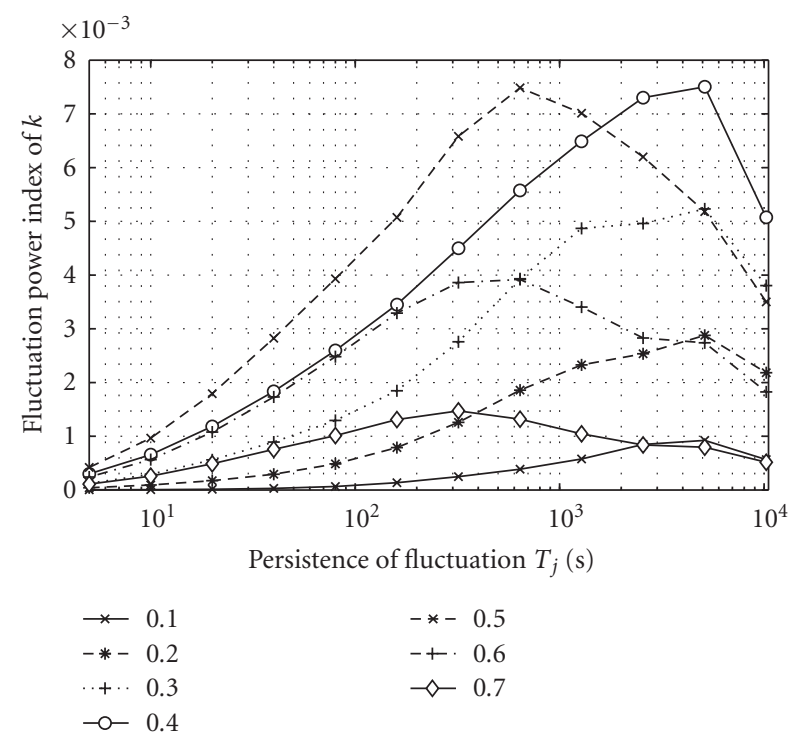

(a) Year 1: data from 362 days from May 14, 2001 to May 31, 2002 .

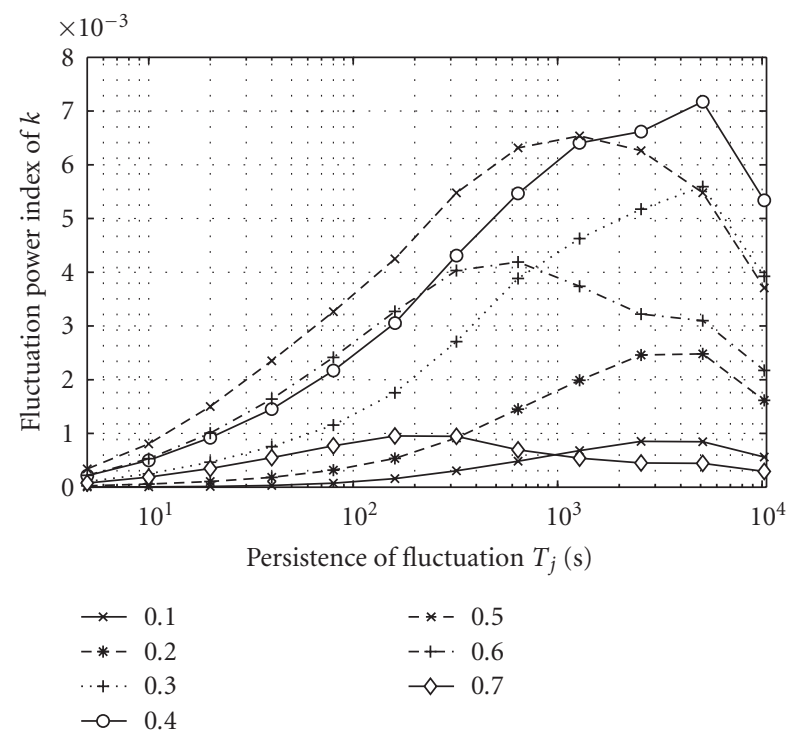

(b) Year 2: data from 359 days from June 1, 2002 to May 31, 2003.

FIgURE 3: Fluctuation power index $c f_{p}$ of clearness index as a function of persistence $T_{j}=S_{j} \Delta T$, annual mean values; legend: class of daily mean clearness index $\bar{k}$.

Figure 3 shows the annual mean values of $c f_{p}$ as a function of persistence of the fluctuation for two successive years. The parameter $\bar{k}$ specifies different classes of daily mean clearness index, and it can be interpreted as a measure for the average cloudiness during a day of the respective class.

For shorter persistence, the mean value of $c f_{p}$ is generally lower than for longer persistence. With an exponentially increasing persistence $S_{j}$, it increases slightly faster than linearly up to a local maximum. 
Clearly, for very low $\bar{k}$ (overcast sky) and very high $\bar{k}$ (slightly clouded to clear sky), the fluctuation power index is low. As expected, it takes maximum values for sky conditions with scattered clouds with $0.3 \leq \bar{k} \leq 0.6$. A local maximum is visible around a persistence between 300 up to 700 seconds, indicating that this range of fluctuations is especially significant for network planning accounting for PV power. The local maxima visible at 5000 seconds indicate global changes of the weather conditions during several hours, for example, from clear sky in the morning to cloudy in the afternoon. Moreover, they are influenced by boundary effects. Obviously, these maxima have no significant meaning with regard to short fluctuations of the solar irradiance.

The striking similarity of Figures 3(a) and 3(b) indicates a significance of the mean $c f_{p}$ as an estimator for the characteristic pattern of irradiance fluctuations under the given climate. The steeper, but narrower local maximum at $\bar{k}=0.5$ in Figure 3(a), originates from a relatively more frequent occurrence of days with steep clear-cloudy transitions in Year 1 in comparison to Year 2 [10].

For further statistical analysis a wider data base is required. The data from Leuven and also from other sites are analyzed in detail on the background of solar energy meteorology in [10]. The high-resolution measurements of solar radiation data in Leuven are still ongoing.

\subsection{Application to distribution systems}

Output fluctuations from PV can lead to energetic imbalances in microgrids and to voltage fluctuations at the endpoints of long radial low-voltage cables. In order to buffer such fluctuations, especially, the application of double-layer capacitors, also referred to as supercapacitors, has been proposed [30, 31].

Power system parameters such as voltage or power reflect fluctuations in the clearness index. The $c f_{p}$ curves can be calculated for clearness index or for PV output power. Here, we assume a linear PV system model which means that, with proper normalization, the $c f_{p}$ curves for clearness index and PV output power are identical. The capacity of energy buffers, necessary for smoothing out power fluctuations introduced by a PV system can then be determined by means of $c f_{p}$ curves as in Figure 3. The curves exhibit local maxima of $c f_{p}$ for $\bar{k}$ values between 0.3 and 0.6 , which corresponds to sky conditions with scattered clouds. The average energy $E_{B}\left(T_{j}\right)$, freed and bound again during one such fluctuation of persistence $T_{j}$, can be derived from the fluctuation power index at $T_{j}$ :

$$
E_{B}\left(T_{j}\right)=T_{j} \sqrt{c f_{p}\left(T_{j}\right)}
$$

where $E_{B}\left(T_{j}\right)$ can be interpreted as the energy to be buffered during $T_{j}$ in order to compensate for fluctuations of this persistence and shorter, with a severity, characterized by the associated $c f_{p}$. The persistence $T_{j}$ in seconds is derived from $S_{j}$ by multiplication with the sampling period $\Delta T$.
An alternative to short-term storage for the mitigation of power fluctuations is demand-side management. In that case, the operation of noncritical loads such as, for example, a fridge, is slightly shifted in time in order to bridge a period of low power supply from the PV system.

Although both measures differ significantly regarding practical implementation, buffering and demand-side management technically have the same effect. In both cases, the consumption of a specified amount of energy during a certain time is postponed to a later moment when sufficient excess energy is available. In both cases, the maximum energy to be shifted as well as the shifting time are subject to practical limitations.

Further, and more detailed, examples for the application of this analysis have been presented in $[8,9]$.

\section{CONCLUSIONS}

Short fluctuations in solar irradiance are a serious drawback for the large-scale application of photovoltaics embedded in the power distribution grid. For the analysis of such fluctuations, signal processing methods should be applied in order to provide a solid mathematical basis for all subsequent conclusions regarding the impact of photovoltaics on the power system. This should enable power system operators and energy supply companies to choose appropriate measures regarding demand side management, energy storage, or upgrading of equipment, based on such analysis methods.

The stochastic fraction of solar irradiance introduced by atmospheric turbidity and moving clouds is represented by the clearness index. The probability distribution of the clearness index is generally determined by its mean value, independent of the season. Hence, statistical analysis of solar irradiance fluctuations must focus on the instantaneous clearness index.

The parameters of interest are the amplitude of the fluctuations, their persistence in time, and their frequency of occurrence rather than their exact time of occurrence. Therefore, a spectral analysis of the fluctuating time series of the clearness index is much more appropriate than a timedomain approach.

Since fluctuations introduced by moving clouds exhibit no periodicity, the power spectral density based on harmonic analysis is not suited for the treatment of such time series. A better parameter for the description of the relevant fluctuations is the local spectral density of the fluctuating time series, interpreted as realizations of a locally stationary wavelet process.

Wavelet periodograms as an estimator for the local spectral density allow an assessment of the amplitude of fluctuations classified by their characteristic persistence. For this analysis, the Haar wavelet should be applied since it approximates well the bimodal character of the clearness index.

The fluctuation power index $\left(c f_{p}\right)$ and fluctuation energy index $\left(c f_{e}\right)$ have been introduced as the mean power of the fluctuating time series, respectively, the energy associated with one fluctuation, both for each scale. The annual 
averages of the fluctuation power index for two successive years exhibit a very close agreement, indicating some significance as an estimator for the characteristic pattern of shortterm irradiance fluctuations in the specific climate. The application of the fluctuation power index has briefly been sketched for the sizing of energy buffers in microgrids and distribution feeders with a high share of photovoltaic generation.

In the future, further statistical analysis is necessary, based on a much wider base of empirical data. The proposed method that has proven valuable for the processing of nonstationary stochastic signals in many other fields, is best suited also for the systematic analysis of fluctuations in solar irradiance.

\section{ACKNOWLEDGMENTS}

This work has been carried out at Katholieke Universiteit Leuven as a part of the first author's Ph.D. dissertation. It was financed by IMEC vzw, Leuven, in the framework of the IMEC-K. U. Leuven Project 1996-2001/AO602, by the European Commission under Contract no. ENK5-CT-200100522 (DISPOWER), and by the Flemish region under Contract no. IWT-GBOU 010055. The authors thank H. Braunisch and J. Simoens for support and suggestions in the field of signal processing and wavelets, and J. Appelbaum and $\mathrm{H}$. Suehrcke for their support, criticism, and suggestions regarding the stochastic behavior of the instantaneous clearness index.

\section{REFERENCES}

[1] M. Misiti, Y. Misiti, G. Oppenheim, and J.-M. Poggi, "Décomposition par ondelettes et méthodes comparatives: étude d'une courbe de charge électrique," Revue de Statistique Appliquée, vol. 42, no. 2, pp. 57-77, 1994.

[2] M. E. Degaudenzi and C. M. Arizmendi, "Wavelet-based fractal analysis of electrical power demand," Fractals, vol. 8, no. 3, pp. 239-245, 2000.

[3] S. Santoso, E. J. Powers, and W. M. Grady, "Power quality disturbance data compression using wavelet transform methods," IEEE Transactions on Power Delivery, vol. 12, no. 3, pp. 12501256, 1997.

[4] S. Santoso, W. M. Grady, E. J. Powers, J. Lamoree, and S. C. Bhatt, "Characterization of distribution power quality events with Fourier and wavelet transforms," IEEE Transactions on Power Delivery, vol. 15, no. 1, pp. 247-254, 2000.

[5] P. Pierz and E. Rosołowski, "Analysis and reconstruction of the shape of the voltage sag using wavelet transform," in Fachtagung Elektrische Energiewandlungssysteme, pp. 74-77, Magdeburg, Germany, March 2002.

[6] T. Croes, C. Gherasim, J. Van Den Keybus, J. Ghijselen, J. Driesen, and R. Belmans, "Power measurement using the wavelet transform of analytic signals," in Proceedings of the 11th International Conference on Harmonics and Quality of Power, pp. 338-341, Lake Placid, NY, USA, September 2004, CD-ROM.

[7] C.-H. Lee, Y.-J. Wang, and W.-L. Huang, "A literature survey of wavelets in power engineering applications," Proceedings of the National Science Council, Republic of China, Part A, vol. 24, no. 4, pp. 249-258, 2000.

[8] A. Woyte, V. V. Thong, R. Belmans, and J. Nijs, "Voltage fluctuations on distribution level introduced by photovoltaic systems," IEEE Transactions on Energy Conversion, vol. 21, no. 1, pp. 202-209, 2006.

[9] A. Woyte, M. Bodach, R. Belmans, and J. Nijs, "Power fluctuations in micro-grids introduced by photovoltaics: analysis and solutions," in Proceedings of the 2nd European PV-Hybrid and Mini-Grid Conference, pp. 449-454, Kassel, Germany, September 2003.

[10] A. Woyte, R. Belmans, and J. Nijs, "Fluctuations in instantaneous clearness index: Analysis and statistics," Solar Energy, vol. 81, no. 2, pp. 195-206, 2007.

[11] G. P. Nason and B. W. Silverman, "The stationary wavelet transform and some statistical applications," in Wavelets and Statistics, A. Antoniadis and G. Oppenheim, Eds., vol. 103 of Lecture Notes in Statistics, pp. 281-299, Springer, New York, NY, USA, 1995.

[12] G. P. Nason, R. von Sachs, and G. Kroisandt, "Wavelet processes and adaptive estimation of the evolutionary wavelet spectrum," Journal of the Royal Statistical Society. Series B, vol. 62, no. 2, pp. 271-292, 2000.

[13] S. Van Bellegem and R. von Sachs, "Locally adaptive estimation of sparse evolutionary wavelet spectra," Discussion Paper 310, Institut de Statistique, Université Catholique de Louvain, Louvain-la-Neuve, Belgium, May 2003, http://www. stat.ucl.ac.be/ISpersonnel/vanbelle/res.html.

[14] E. C. Kern Jr., E. M. Gulachenski, and G. A. Kern, "Cloud effects on distributed photovoltaic generation: slow transients at the Gardner, Massachusetts photovoltaic experiment," IEEE Transactions on Energy Conversion, vol. 4, no. 2, pp. 184-190, 1989.

[15] W. Jewell and R. Ramakumar, "The effects of moving clouds on electric utilities with dispersed photovoltaic generation," IEEE Transactions on Energy Conversion, vol. 2, no. 4, pp. 570576, 1987.

[16] H. Beyer, A. Hammer, J. Luther, J. Poplawska, K. Stolzenburg, and P. Wieting, "Analysis and synthesis of cloud pattern for radiation field studies," Solar Energy, vol. 52, no. 5, pp. 379390, 1994.

[17] K. Otani, J. Minowa, and K. Kurokawa, "Study on areal solar irradiance for analyzing areally-totalized PV systems," Solar Energy Materials and Solar Cells, vol. 47, no. 1-4, pp. 281-288, 1997.

[18] M. Iqbal, An Introduction to Solar Radiation, Academic Press, Don Mills, Ontario, Canada, 1983.

[19] A. Skartveit and J. A. Olseth, "The probability density and autocorrelation of short-term global and beam irradiance," Solar Energy, vol. 49, no. 6, pp. 477-487, 1992.

[20] H. Suehrcke and P. G. McCormick, "The frequency distribution of instantaneous insolation values," Solar Energy, vol. 40, no. 5, pp. 413-422, 1988.

[21] P. Bendt, M. Collares-Pereira, and A. Rabl, "The frequency distribution of daily insolation values," Solar Energy, vol. 27, no. 1, pp. 1-5, 1981.

[22] J. M. Gordon and T. A. Reddy, "Time series analysis of hourly global horizontal solar radiation," Solar Energy, vol. 41, no. 5, pp. 423-429, 1988.

[23] K. G. T. Hollands and R. G. Huget, "A probability density function for the clearness index, with applications," Solar Energy, vol. 30, no. 3, pp. 195-209, 1983. 
[24] B. H. Y. Liu and R. C. Jordan, "The interrelationship and characteristic distribution of direct, diffuse and total solar radiation," Solar Energy, vol. 4, no. 3, pp. 1-19, 1960.

[25] R. R. Coifman and D. L. Dohono, "Translation-invariant denoising," in Wavelets and Statistics, A. Antoniadis and G. Oppenheim, Eds., vol. 103 of Lecture Notes in Statistics, pp. 125-150, Springer, New York, NY, USA, 1995.

[26] S. Mallat, A Wavelet Tour of Signal Processing, Academic Press, San Diego, Calif, USA, 1999.

[27] J.-C. Pesquet, H. Krim, and H. Carfantan, "Time-invariant orthonormal wavelet representations," IEEE Transactions on Signal Processing, vol. 44, no. 8, pp. 1964-1970, 1996.

[28] I. Daubechies, Ten Lectures on Wavelets, SIAM, Philadelphia, Pa, USA, 1992.

[29] A. V. Oppenheim and R. W. Schafer, Discrete-Time Signal Processing, Prentice-Hall, Englewood Cliffs, NJ, USA, 1989.

[30] M. Bodach, "Short term power backup in PV systems with ultra capacitors," in Proceedings of the 17th European Photovoltaic Solar Energy Conference and Exhibition (EUPVSEC '01), pp. 518-519, Munich, Germany, October 2001.

[31] M. H. Rahman, J. Nakayama, K. Nakamura, and S. Yamashiro, "An intelligent grid-connected PV-ECS system with load leveling function," in Proceedings of the 3rd IASTED International Conference on Power and Energy Systems (EUROPES '03), pp. 75-80, Marbella, Spain, September 2003.

Achim Woyte received the Electrical Engineering degree from the University of Hannover (Germany) in 1997 and the Ph.D. degree in engineering from the Katholieke Universiteit Leuven (Belgium) in 2003. He is coauthor of more than 50 scientific publications. He spent half a year working and studying in Venezuela and Italy. He also worked for over three years in electroheat and high-voltage engineering at the Univer-

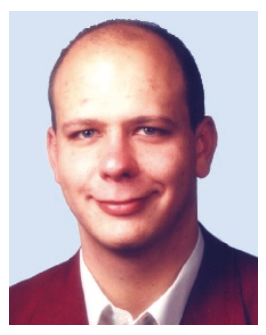
sity of Hannover. He worked out a Master's thesis at the Solar Energy Research Institute (ISFH) in Hameln/Emmerthal (Germany), where he assessed grid-connected photovoltaic systems with regard to the issue of partial shadowing. For his Ph.D. dissertation, he investigated the grid integration of photovoltaic systems including the assessment of photovoltaic components. At the beginning of 2004, he joined the Policy Studies Department of the Brusselsbased consultant 3E. There, he coordinates policy-related projects in renewable energy technology. He also performs research and engineering regarding the integration of electricity from renewable sources into power systems and markets.

Ronnie Belmans received the M.S. degree in electrical engineering in 1979 and the Ph.D. degree in 1984, both from the K. U. Leuven, Belgium, the Special Doctorate in 1989, and the Habilitierung in 1993, both from the RWTH, Aachen, Germany. Currently, he is a Full Professor with the K. $\mathrm{U}$. Leuven, teaching electric power and energy systems. His research interests include technoeconomic aspects of power systems, power quality, and distributed generation. He is also Guest Professor at Imperial College of Science, Medicine and Technology, London, UK. Since June 2002, he is Chairman of the board of directors of ELIA, the Belgian transmission grid operator.
Johan Nijs received the Electrical Engineering University degree in 1977, the Ph.D. degree in applied sciences in 1982, and the degree of Master of Business Administration in 1994, all from the Katholieke Universiteit Leuven, Belgium. After having worked, respectively, at Philips (Belgium), K. U. Leuven (Belgium), and I.B.M. Thomas J. Watson Research Center (NY, USA), he joined in 1984 the Interuniversity Micro Electron-

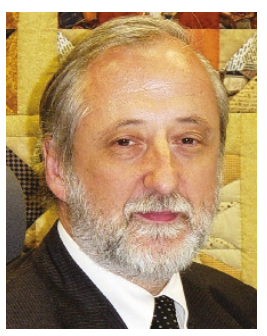
ics Center (IMEC) in Leuven, Belgium, where he became Group Leader of the silicon materials and solar cell activities. In 2000, he became an Associate Vice President and Department Director of the Packaging, MEMS and Photovoltaics Department. From 1990 onwards, he has also been appointed Part-Time Associate Professor at the K. U. Leuven. From 1995 till 1997, he also part-time managed Soltech in Leuven, Belgium (Soltech commercializes photovoltaic energy systems). Since December 2001, his main activity has been the set-up of the IMEC spin-off company PHOTOVOLTECH in Tienen, Belgium, which he fully joined in January 2003 as General Manager. Photovoltech manufactures photovoltaic solar cells and modules. 